\title{
Linear Robot as The Approach towards Individual Abdominal Ultrasound Scanning in Developing Countries?
}

\author{
Christina Pahl
}

\begin{abstract}
In order to improve comfort in abdominal ultrasound scanning, reduce operator dependency and increase image resolution, a novel method for automatic ultrasound cervix scanning, especially for developing countries, has been developed. To determine the reference scanning point for customized diagnosis procedures, personalized scanning algorithms based on umbilicus detection are utilized. The setup consists of three linear motors controlled by an ATmega328 microcontroller and is based on real-time data of an IR sensor as feedback information. For testing purposes 10 subjects were scanned using a robotic platform. Robotically acquired data were compared with manually taken ultrasound images. Safety and image quality have been evaluated and approaches developed to counteract mentioned deficits. A correlation analysis as well as image quality classification between histograms and probe pressure image data was performed. Results show an automated method for personalized ultrasonography with a maximum accuracy of the defined scanning reference being $\mathbf{9 8 . 4} \%$ for one dimension. It can be concluded that simple image processing techniques like 3D reconstruction and merging in combination with robotically guided ultrasound probe motion are highly efficient to counteract existing ultrasonography deficits. Future works consider the application of automated ultrasonography on related medical fields.
\end{abstract}

Keywords-linear robot, merging, personalized ultrasonography, probe pressure, safety.

\section{INTRODUCTION}

Referring to the most recent cancer statistics provided on the World Health Organisation (WHO) database, maintained by the International Agency of Research on Cancer (IARC), cervical cancer represents the second most common cancer in women worldwide. The international incidence rates of cervical cancer show that almost $80 \%$ of cervix cancer cases occur in low-income countries [1]. Therefore, in developing countries like Malaysia, cervical cancer has become a serious problem and is currently part of discussions in health Politics [2]. According to WCRF International the new incidence rates worldwide of colorectal cancer show that with a number of 614,000 in the year of 2012, this cancer represents nearly $10 \%$ of all international cancer cases [3]. It can be seen that cancer types occurring in the abdominal area are dominating in the list of all cancers in females as well as males worldwide.

This work was supported by Universiti Teknologi Malaysia under Grant 03G12. Manuscript received Nov. 12 2015, revised Dec. 18, 2015 C. Pahl is with the Biomechatronics Group, Technische Universität Ilmenau, 98693 Ilmenau, Germany (phone: +49 3677-694690; e-mail: christina.pahl@tu-ilmenau.de) as well as with IJN-UTM Cardiovascular Engineering Centre, University Technology Malaysia, Skudai, 81310, Malaysia.
In order to decrease the number of abdominal cancer cases by improving the diagnosis of these malignances, an approach on the automation of ultrasonography based on a personalized setup [4] consisting of a starting point with the lowest risk factors and costs will be presented here [5]. Although, ultrasonography is highly operator dependent and provides a low contrast resolution [6], it is used to evaluate the size and extent of tumors [7]. Among existing imaging techniques, ultrasound is preferred in the examination of the abdomen. It is affordable compared to MRI and CT [8], widely available, noninvasive, non-ionizing [9] and painless [10]. Ultrasonography is a medical imaging technique that uses high frequency sound waves [11] and their echoes [12]. The technique is similar to the echolocation used as SONAR by submarines [13].

The machine displays the distances and intensities of the echoes on the screen [14] forming a two dimensional [15] image [16]. Table I shows a basic comparison of existing technologies for abdominal cancer visualization. However, the use of ultrasound in medical examinations encompasses some limitations. A major drawback of ultrasonography is high operator dependency [17]. Since image acquisition is complex, diagnostic accuracy in ultrasound is directly related to skills, training and experience of the operator [18].

Moreover, experience is highly required in analyzing and interpreting the anatomical appearance of malign structures [19]. Each operator may interpret one and the same ultrasound image of cancer differently [20]. Concerning the limited capabilities of ultrasound in cervix imaging, it is important to develop alternative approaches to remove the current disadvantages. Autonomous systems can help in minimizing operator dependency, to increase reproducibility and thus to increase comparability of image data for further diagnosis. One approach towards this represents the usage of linear motors performing the abdominal cervix scan. Its high accuracy in moving the transducer probe and stability in keeping a desired pose [21] allow to acquire highly comparable data [22]. Currently, there are no automatic systems developed for abdominal ultrasound diagnostics like the proposed system [23].

TABLE I

COMPARISON OF CT, MRI AND ULTRASOUND ( $\mathrm{X}=\mathrm{LOW}$, $\mathrm{XX}=\mathrm{MEDIUM}, \mathrm{XXX}=\mathrm{HIGH} ; \mathrm{N}=\mathrm{NO}, \mathrm{Y}=\mathrm{YES})$

\begin{tabular}{lllll}
\hline \hline Modality & Invasiveness & Cost & Time & Sensitivity \\
\hline CT & $\mathrm{N}$ & $\mathrm{XX}$ & $\mathrm{XX}$ & $\mathrm{XX}$ \\
MRI & $\mathrm{N}$ & $\mathrm{XXX}$ & $\mathrm{XXX}$ & $\mathrm{XXX}$ \\
$\mathrm{US}$ & $\mathrm{N} / \mathrm{Y}$ & $\mathrm{X}$ & $\mathrm{X}$ & $\mathrm{X}$ \\
\hline \hline
\end{tabular}




\section{A. Ultrasound Safety Considerations}

Besides its apparently excellent safety record, medical ultrasonography still exposes energy in the organic tissues [24]. There appear biological effects if high power in combination with long exposure time is applied. Due to its thermal effects, safe usage of this imaging method should be taken into consideration in clinical applications. In order to correlate potential ultrasound effects with exposure, a characterization of the ultrasound beam is essential [25]. In this work, brightness mode (B-mode) imaging will be applied to acquire $2 \mathrm{D}$ ultrasound images of the human cervix.

\section{B. Effects of Ultrasound Imaging}

In contrast to Doppler diagnosis, B-mode imaging has not demonstrated to produce harmful temperature rises in the body [26]. However, a basic approach towards safe use of ultrasound is the as low as reasonably achievable (ALARA) principle. Two indices are introduced here: mechanical index (MI) and thermal index (TI). Whereas, MI is associated with the degree of cavitation bio-effects, TI indicates the tissue heating bio-effect. Cavitation is a non-thermal bio-effect, which takes place when small gas bubbles oscillate, increase in size, and collapse due to the influence of ultrasonic field [27]. Thermal effects indicated by TI, may result in the increase of tissue extensibility, blood flow but also reduction in joint stiffness and muscle spasm. Fig. 1 follows the guidelines for the safe use of diagnostic ultrasound equipment provided by the British Medical Ultrasound Society (BMUS) and shall give an overview about the relation between recommended scanning time and metric TI. It can be obtained that with increasing TI, the recommended scanning time decreases. At this point it can be concluded that a compromise between scanning time and TI needs to be defined in order to achieve the highest possible image quality with regards to safety requirements. Therefore, a TI value between 1.5 and 2.5 will be used.

\section{Intensity and Exposure Time}

A typical diagram is illustrated in Fig. 2 showing the intensity of the ultrasound beam with given frequency against the exposition time. The duration used image the inner organs of the human body is restricted by the time the ultrasound is exposed to the body.

In Fig. 2 safe, critical and unsafe area are indicated. It can be seen that with increasing scanning time, the frequency needs to be adjusted in order to stay inside the area of safe usage of the clinical ultrasound.

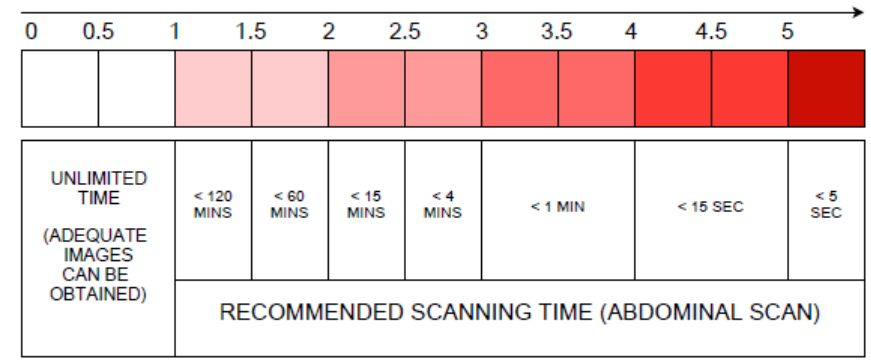

Fig. 1. Thermal Index and Recommended Scanning Time

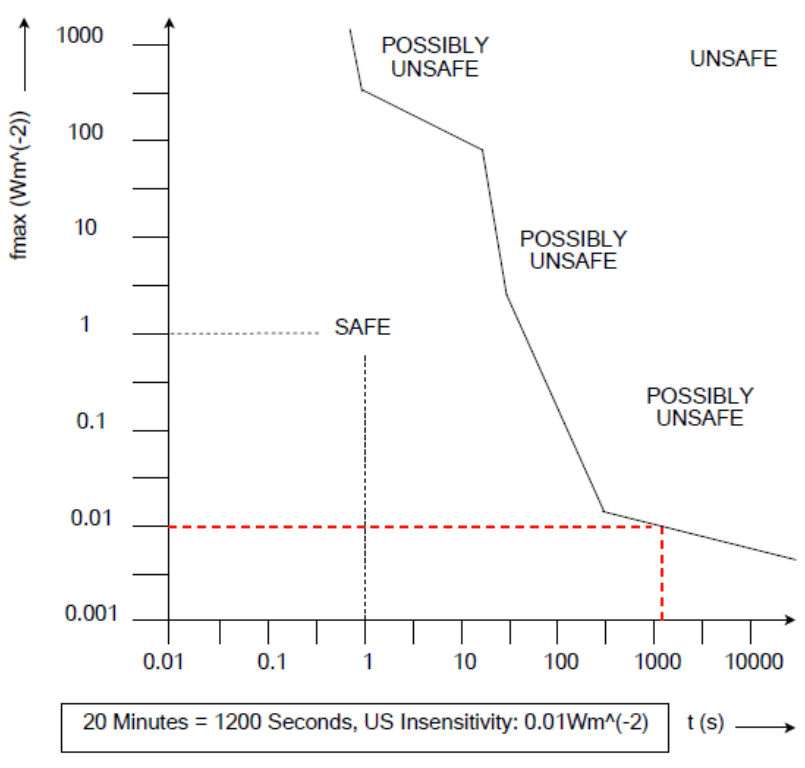

Fig. 2. Ultrasound Safety According to Frequency and Time

In Fig. 2 guidelines of the American Institute of Ultrasound in Medicine (AIUM) regarding the ultrasound intensity and allowed exposure time are shown. The beam frequency should be high for lateral resolution but depends on the depth of the organs, which need to be scanned. In order to image the cervix with the highest duration for reconstruction purposes and panoramic views, the highest scanning time possible for best possible results is required. Being here restricted to safety specifications, a compromise needs to be made. Therefore, a maximum intensity of $0.01 \mathrm{Wm}^{2}$ is chosen to be applied. This is illustrated by the dashed red line in Fig. 2.

\section{DESIGN AND IMPLEMENTATION}

It is important to develop alternative approaches to minimize the current disadvantages in ultrasound imaging due to the limited capabilities of ultrasound in cancer imaging. The suggested setup for automatic ultrasound scanning shall be cost-effective, stable and provide a high movement resolution. An algorithm was developed and allows operator independent detection of the umbilicus on the abdominal surface. The control scheme of the developed system can be seen in Fig. 3. It can be obtained that three motors receive data from the Atmega328 Microcontroller. Furthermore, the gear used to transmission the movement to the linear motion system and the motors used for the generation of motion proportional to the signal of the drivers. The electrical part consists of drivers, which provide voltage according to data they receive from the controller. The last part involves computing. The three motors satisfy the movements in three dimensions. These motors also move the position of the Infrared (IR) sensor.

This sensor sends data to the microcontroller, which analyzes the values according to the algorithm input from the computer and regulates subsequently the position of the sensor by correcting the movement commands of the motors [28]. The ultrasound machine used here was the TOSHIBA APLIO-MX ultrasound system. 


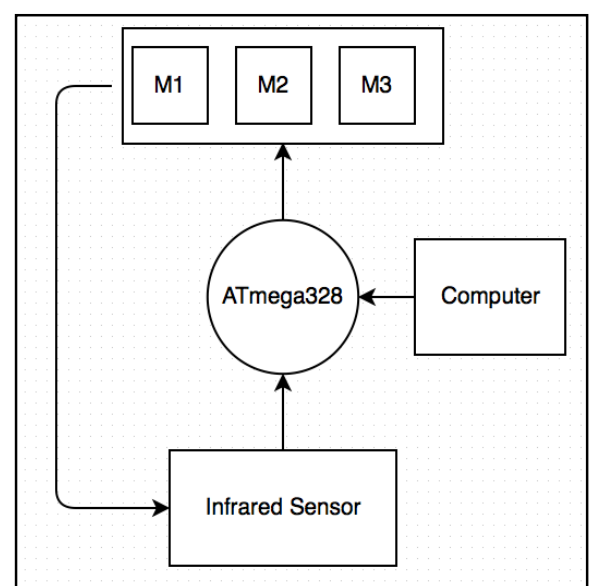

Fig. 3. Control unit of personalized ultrasound scan for automatic umbilicus localization using IR technology.

\section{A. Characteristics for Abdominal Scanning}

The IR sensor being the main part of this setup is attached to the transabdominal ultrasound probe and its mechanic holder. The setup was tested using two phantoms and 10 subjects. The characteristics of the subjects can be seen in Table II. Adult subjects, listed with the capital letter $S$ and a following number, were scanned. All of the subjects were female. The youngest subject was 21 years old, whereas the oldest was documented with 36 years. The letter $A$ represents the age. Here, $C$ refers to the circumference of the abdomen. It is important since the abdominal detection for the umbilicus is different in the case large abdominal tissue is available or if the opposite case holds true. The letter $H$ represents height. The letter $W$ referring to weight can be seen as an indicator for the body mass in relation to height and therefore and index for abdominal mass. $U V$ refers to the distance between umbilicus and pubic bone.

\section{B. Umbilicus Detection}

Fig. 4 displays the algorithm for the motor and driver control in order to localize the umbilicus. The algorithm consists of eight decision steps. The starting point marks the position of the three motors with $X_{i}, Y_{i}$ and $Z_{i}$ being here zero indexed.

TABLE II

CHARACTERISTICS OF SCANNED SUBJECTS

\begin{tabular}{llllll}
\hline \hline & A & C & H & W & UV \\
& {$[$ YEARS $]$} & {$[\mathrm{CM}]$} & {$[\mathrm{CM}]$} & {$[\mathrm{KG}]$} & {$[\mathrm{CM}]$} \\
\hline S1 & 36 & 79 & 154 & 59 & 18 \\
S2 & 29 & 76 & 148 & 49 & 20 \\
S3 & 28 & 74 & 152 & 61 & 19 \\
S4 & 26 & 71 & 164 & 57 & 26 \\
S5 & 24 & 69 & 154 & 52 & 23 \\
S6 & 23 & 63 & 169 & 58 & 26 \\
S7 & 23 & 87 & 158 & 54 & 19 \\
S8 & 22 & 73 & 147 & 50 & 21 \\
S9 & 21 & 73 & 158 & 58 & 21 \\
S10 & 21 & 75 & 164 & 65 & 23 \\
\hline \hline
\end{tabular}

Subsequently, destination and data acquisition represents the approximation of the umbilicus based on personalized values calculated for each subject on the basis of the parameters from Table II according to (1).

$$
I_{S A}=A+C+H \times W+U V^{2},
$$

where $I_{S A}$ represents the individual scanning area defining the extreme points of the frame of probe movement as shown Values for $I_{S A}$ in this setup range between 7000 and 12000, where 1000 points define a $1 \mathrm{~cm}$ wider scan range leading to a 4 times bigger scan window for subject $S 10$ compared to $S 2$ for example. The direction and pulse are subsequently set.

Meanwhile, to the movement of the transducer and therefore the IR sensor, the values for the sensor are sent to the microcontroller and compared with experimentally predefined thresholds. Experiments show that a threshold between $360 \mathrm{mV}$ and $380 \mathrm{mV}$ delivers the required results for the detection of the umbilicus. In the case that the values differ from the threshold, the motor will be continuously moved until the values overlap and the motors stops automatically. The third method shows the approximation of the umbilicus by orbiting the in $90^{\circ}$ angulations defining a small circle at the starting point and resulting in bigger circles in the end. This approach showed the highest rate of success in umbilicus approximation and was therefore used in this setup.

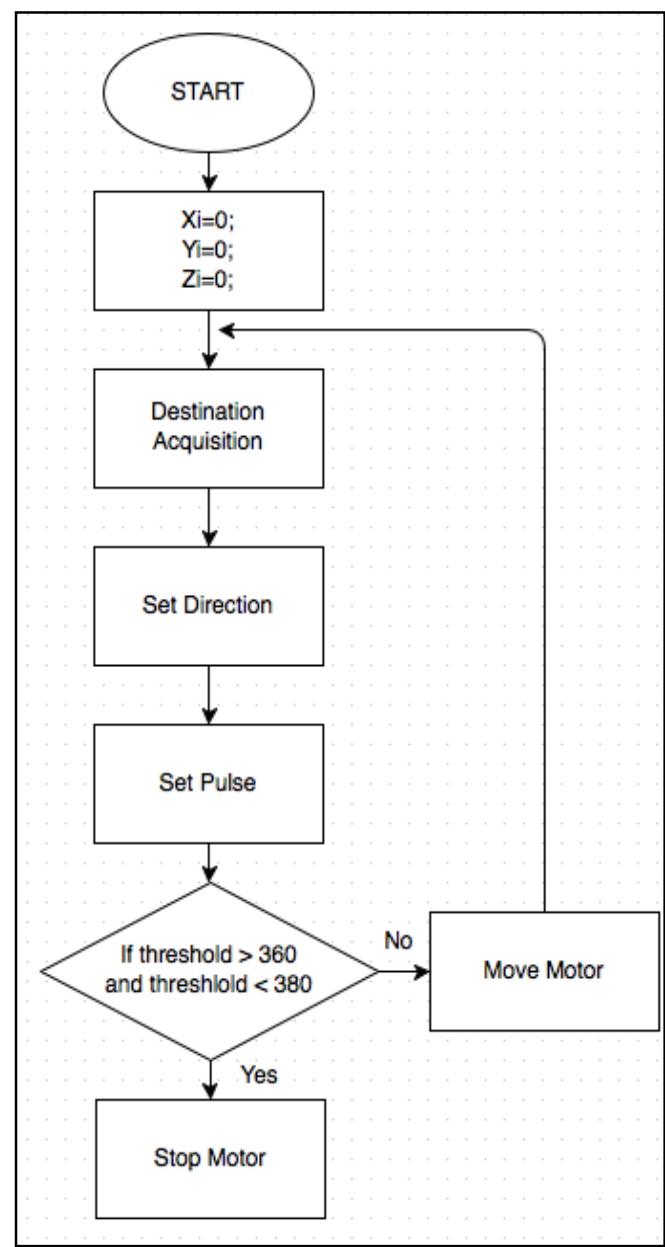

Fig. 4. Side and top view of the setup for the automatic and personalized ultrasound scans. 

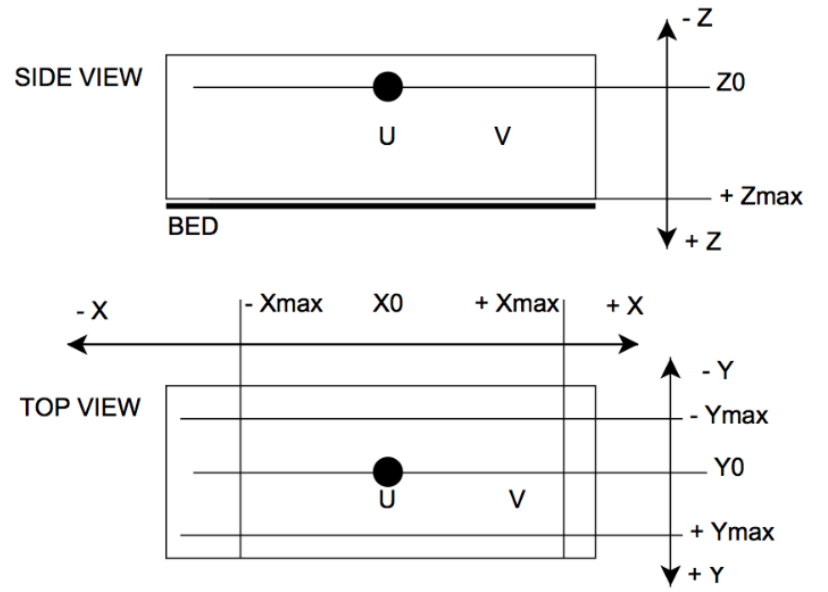

Fig. 5. Side and top view of the setup for the automatic and personalized ultrasound scans.

\section{System Setup}

The mechanical movement extension restricts the automatic scan [29]. Fig. 5 shows the setup for the reference scan using operator dependent parameters. There are two subfigures provided. The first one displays the side view, whereas the second one shows the top view. The allocation of the parameters is displayed. The letter $U$ represents the umbilicus, whereas $V$ stands for the end of the pubic bone. Moreover, Fig. 5 shows the setup for the detection of the umbilicus shown in side and front view. An IR sensor was attached to the probe holder. The voltage output of this sensor is a function of the distance between the sensor and the reflected IR waves. Because of the differing position of the sensor referring to the probe, a shift of $2.5 \mathrm{~cm}$ needs to be corrected in order to adjust the position of the probe for subsequent scanning. This is done after successful detection. The analog output of the IR sensor was connected to the digital converter of the $\mu \mathrm{C}$ in order to take subsequent distance measurements. On a distance between the sensor and the umbilicus ranging $4 \mathrm{~cm}$ to $20 \mathrm{~cm}$, measurements were performed acquiring approximately 2 Million voltage values.

For this setup, the processor of the PC should be at least $2.66 \mathrm{GHz}$ for the processing of the video grabber device, the Operating System (OS) should be at least Windows 2000 or any other OS like OS X 10.3 or Linux 2.6x. The hard disc should be able to store at least $5 \mathrm{~GB}$, which represents 100 videos captured. The RAM should have a capacity of at least $256 \mathrm{MB}$. Out of these data, a distance between sensor and umbilicus marker of $3 \mathrm{~cm}$ was chosen because this value represents the highest voltage output.

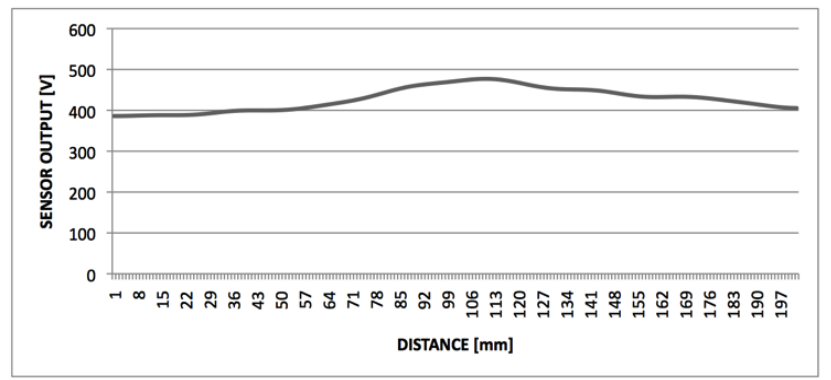

Fig. 6. Side and top view of the setup for the automatic and personalized ultrasound scans.
A squared object with an edge length of $3 \mathrm{~cm}$ was chosen as a marker for the umbilicus. This object was then placed above the umbilicus and used as a marker. The distance between marker and the highest point of the abdomen was set as $3 \mathrm{~cm}$. The subjects were then placed on the patient bed, so that in lying position 5 linear probe motions parallel with the marker in a height of $3 \mathrm{~cm}$ distance from the marker could be performed. Then the subjects were scanned for 15 minutes per session. The time for umbilicus recognition was in the range of seconds. However, the duration depended on the starting point of the transducer probe and the position of the subject respectively. Humans can visually detect the umbilicus. However, in this setup human intelligence for umbilicus recognition will be substituted. Approaches towards Artificial Intelligence (AI) [30] based robotic systems may be considered for classification of data [31]. Fig. 6 shows a typical voltage output for the infrared scanning procedure above the abdomen.

\section{RESULTS AND ANALYSIS}

\section{A. Scanning Safety}

The feature of an emergency button is necessary, since safety for both, human and machine, is important. For the usage of the emergency button a frequency of $360,000 \mathrm{~Hz}$ and a resolution of 8,000 pulses per rotation was chosen.

This ensures, that the probe moves quickly from its actual position to the initial point. This is because any emergency case requires the ability for fast escape. Choosing the lowest resolution is even at the highest frequency too slow. Choosing the highest resolution is also not effective. Thus, a sufficient velocity was chosen according to the characteristics of the motor. This means, that for the actual position of the probe, which is located at the center of the linear robot, a maximum freedom of movement for the scanned subject can be ensured in emergency cases. From Table III it can be concluded, that the emergency button is working with a sufficient velocity according to human reaction capabilities [32]. The values for $X$ and $Y$ are therefore zero, because the emergency button only considers the $Z$-direction and no other directions. The first column is filled with zeros because to the point of time, the probe position is already in its initial point. The time required to set the probe into its initial point ( 2 seconds) is suitable for applications with occurring emergency cases.

\section{B. Stop button}

Coronal and sagittal images of the cervix have been taken. Moreover, the depth of the probe is displayed in Fig. 7. Stop 1 represents soft touch. Whereas, stop 2 refers to medium pressure, stop 3 is already reported to be hard.

TABLE III

EMERGENCY BUTTON RESULTS

\begin{tabular}{|c|c|c|c|}
\hline Condition & $\begin{array}{c}\text { Initialization/ } \\
\text { patient data input }\end{array}$ & $\begin{array}{c}\text { Umbilicus } \\
\text { localization }\end{array}$ & Scanning \\
\hline Distance [mm] & 0 & 0 & 0 \\
X & 0 & 0 & 0 \\
Y & 0 & 157.3 & 208.2 \\
Z & 0 & 1.16 & 1.53 \\
\hline Time [s] & & & \\
\hline
\end{tabular}


The last stop and simultaneous image capturing was performed for pain felt due to the probe depth in the lower abdomen. From image data, it can be seen that there is a correlation between probe depth and image quality. The more deep the probe is pushed into the abdomen, the better artifact causing gases can be pushed away and the closer the cervix can be examined. In order to better understand Fig. 7, the first row will be analyzed. $S 1$ provides images ranging a depth from $0.8 \mathrm{~cm}$ until $4.1 \mathrm{~cm}$. The first stop indicates, that the subject experiences here soft pressure. The image quality is according to the low pressure less good compared to the following images. Moreover, the uterus and the cervix are less clear. In the next step, the stop button was pushed when medium pressure was felt. The depth has a value of $1.6 \mathrm{~cm}$ in this case. The structures of the uterus and consequently the cervix get more obvious. The third stop was pushed when hard pressure was felt on the abdomen. The image provides in this stage already more information and can be regarded as useful. However, the best image could be obtained with a probe depth of $4.1 \mathrm{~cm}$. The details are at this stage more clearly differentiable compared to previous images. The hollow body of the uterus in this image is highly clear. $D$ stands in Fig. 7 for the transducer probe depth measured from the surface of the abdomen.

\section{Probe pressure and depth}

Common transabdominal ultrasound scanning requires the contact pressure between the probe and the subject in order to achieve a good acoustic match. Another test was performed regarding the probe depth. Pressure must be applied evenly in order to get accurate direction of the scan. Therefore, operator dependent scanning is less suitable than automatic for better image results concerning image quality. Table IV shows the allocation of quality values and the pressure applied. The image quality parameters have been determined on the basis of anatomic landmarks used as reference points. Since the correct pressure application of probe can be used to considerably improve the image quality, probe depth was analyzed. Pressure affects the echogenicity of tissue and shortens the distance to cervical structures. All values are based on contrast properties of images by comparing their histograms. Here, it can be obtained, that in three out of ten cases, the highest pressure could not deliver best image data. Excessive pressure, however, can cause discomfort to the subject. It is assumed that too high pressure causes cramping as a protective reaction and thus influences the imaging procedure and its results. On the basis on the analysis results of an experienced sonographer, the image quality of resulting image data could be defined. This analysis considers contrast, boundary of cervix as well as artefacts. Image quality indicators from 1 to 4 were utilized. Number 4 stands for the lowest and number 1 for the highest pressure applied on the abdomen. All these values reflect subjective impressions of ultrasound probe pressure. Detail contrast can have a significant visual impact on the ultrasound image. This is because it emphasizes texture, so that the cervix appears with a higher number of details and more pronounced highlights.

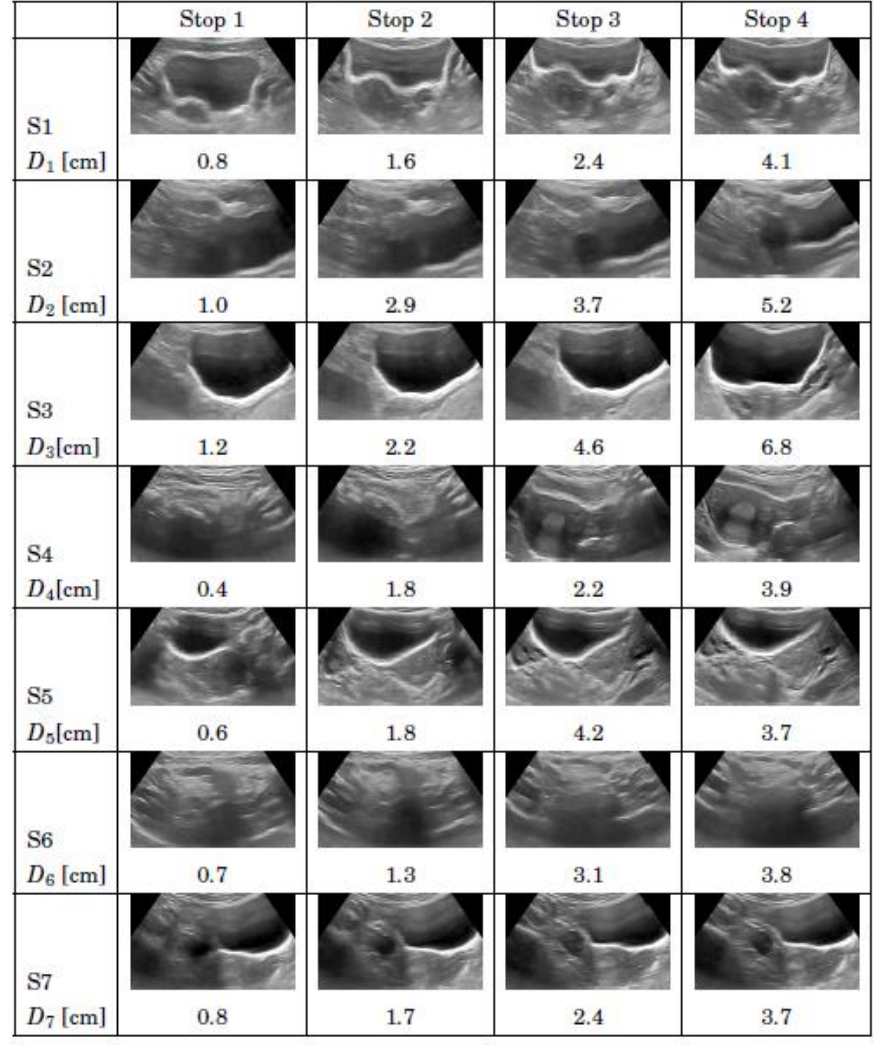

Fig. 7. Stop-button Function and Depth Related Image Quality

Therefore, the histogram approach for image quality analysis was chosen. The histograms for three subjects are displayed in Fig. 8. Subject 5 and 7, show the expecting result, being higher contrast followed by higher pressure. As shown for the last case, the histogram has less contrast for the image under pain than under hard pressure. For each case, soft pressure leads to less contrast in the image, whereas medium pressure increases the contrast. The contrast quality between hard pressure and pain cannot be unambiguously correlated, see Fig. 9. This is case specific and shows the requirement of a pressure feedback using the previously introduced stopbutton.

TABLE IV

Image Quality Compared To Ultrasound Probe Pressure

\begin{tabular}{|l|c|c|c|c|}
\hline Image quality & Pressure & Medium & Hard & Pain \\
\hline S1 & 4 & 3 & 2 & 1 \\
\hline S2 & 4 & 3 & 2 & 1 \\
\hline S3 & 4 & 2 & 1 & 2 \\
\hline S4 & 4 & 3 & 2 & 1 \\
\hline S5 & 4 & 3 & 2 & 1 \\
\hline S6 & 4 & 3 & 2 & 1 \\
\hline S7 & 4 & 3 & 2 & 1 \\
\hline S8 & 4 & 3 & 1 & 2 \\
\hline S9 & 4 & 3 & 1 & 2 \\
\hline S10 & 4 & 3 & 2 & 1 \\
\hline \hline Average & 4 & 2.9 & 1.7 & 1.3 \\
\hline
\end{tabular}




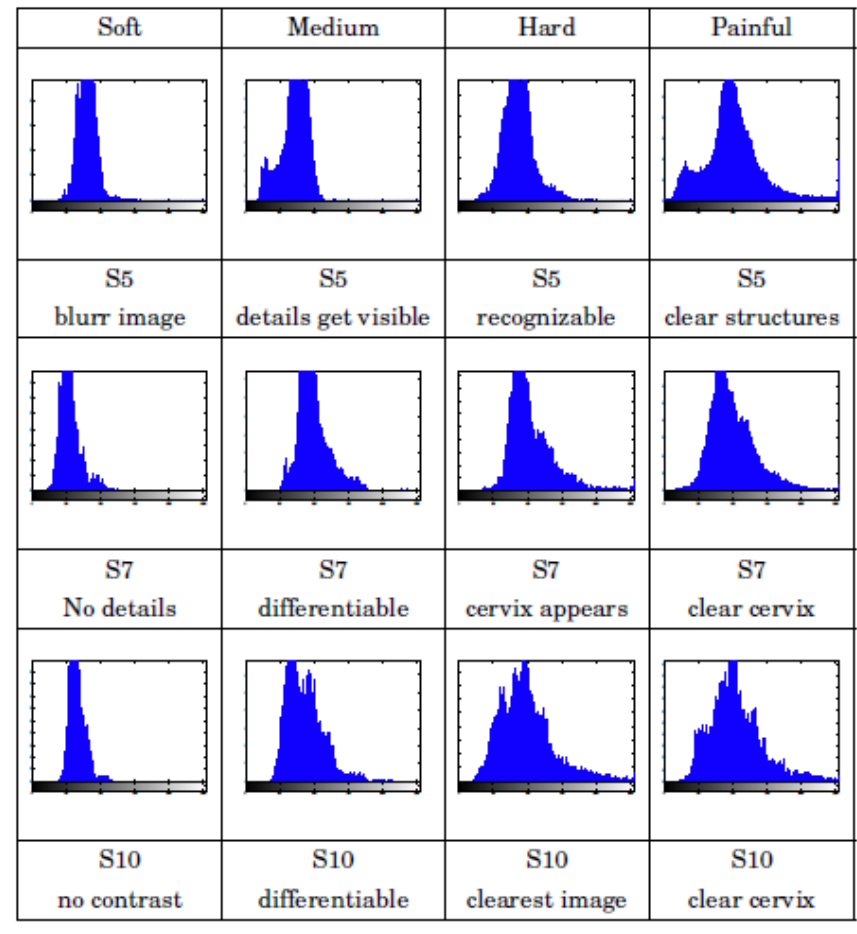

Fig. 8. Histograms of Cervix Ultrasound Images with Different Pressure

In the following, three applications shall be introduced, explaining in how far data, which are acquired by the system, can be used. First, two approaches in improving the image quality on the basis of resolution will be explained. Subsequently, the usage of the scanning system for ultrasound based panoramic views will be explained. Finally, an example using $3 \mathrm{D}$ reconstruction will be shown.

\section{Resolution improvement}

The first approach for improving the ultrasound scanning resolution is based on the idea of combining two or more scans with spatial shifts $\Delta X$ into a single scan as shown in Fig. 10. Merging ultrasound images with restricted detail information leads to the increase of information, which improves the resolution and consequently follows into a higher image quality. For this method, it is useful to have a scanning system like the one developed in this work. Such a system enables the motion of the scanning transducer probe in very small steps, so that the ultrasound probe crystals, which miss the information located in the area of their gaps can be still acquired. Moreover, for this purpose it is important to move the probe with a high accuracy, which can only be provided by machines, currently. Assuming that an ultrasound probe of a length of $6 \mathrm{~cm}$ is covered with 120 crystal elements, the resolution would be $0.5 \mathrm{~mm}$ according to (2).

$$
\text { Probe Resolution }=\frac{60}{120}=0.5
$$

Such a resolution is often not sufficient in providing reliable information on tissues, which could be affected by cancerous cells.

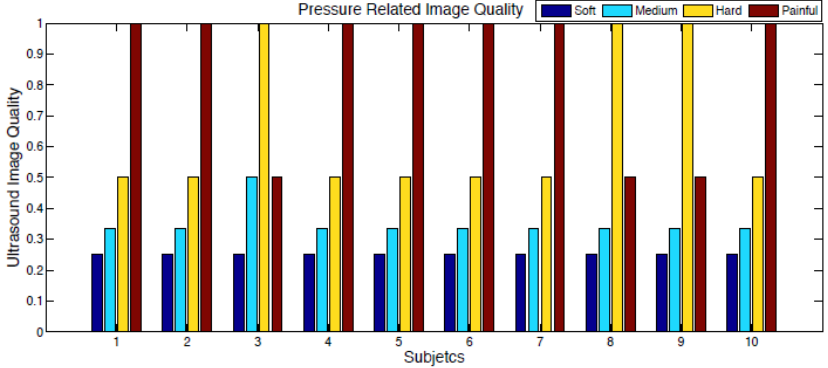

Fig. 9. Relation between Probe Pressure Classes and Image Quality

Therefore, the necessity exists to improve the resolution. Piezoelectric crystals convert electricity into sound and the other way around. The distance between each crystal element is defined as $\Delta X$. A static scan of a phantom containing several elements of the size of the crystals and the distance between them, apparently provides only half of the information originally existing. The motion of the probe in a distance of exactly $\Delta X$ leads respectively to the result that only those phantom details are displayed, which were ignored by the beam due to the gaps. Having two scans now with a shift of the size of the crystal to crystal distance does not provide a better image quality.

Nevertheless, the combination of both images into one image leads to doubled information details. Such a merged image is of higher value for subsequent analysis procedures. Another step towards improving the resolution of ultrasound image data is based on the reconstruction of 3D data from 2D ultrasound images as shown in Fig. 11. Taking into consideration, that an ultrasound phantom consists of single structures being separated from each other in a distance of 20 $\mu \mathrm{m}$, a static scan with the average resolution of $0.5 \mathrm{~mm}$ or even a dynamic scan of a scanning resolution of $20 \mu \mathrm{m}$ and more are not sufficient to differentiate existing line structures.

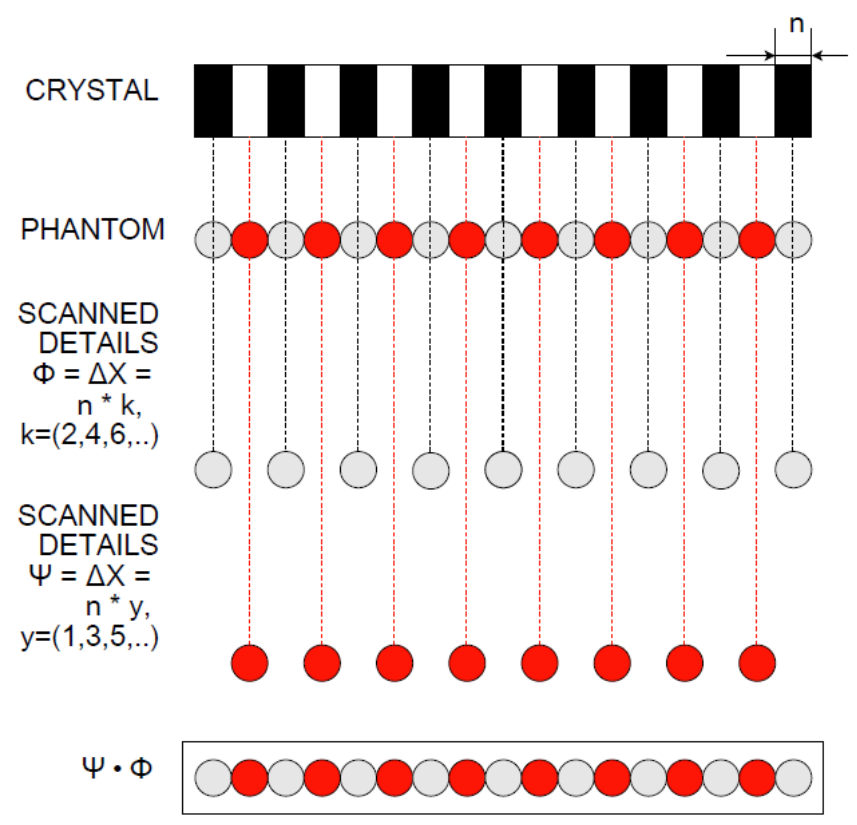

Fig. 10. Resolution Improvement by Merging 


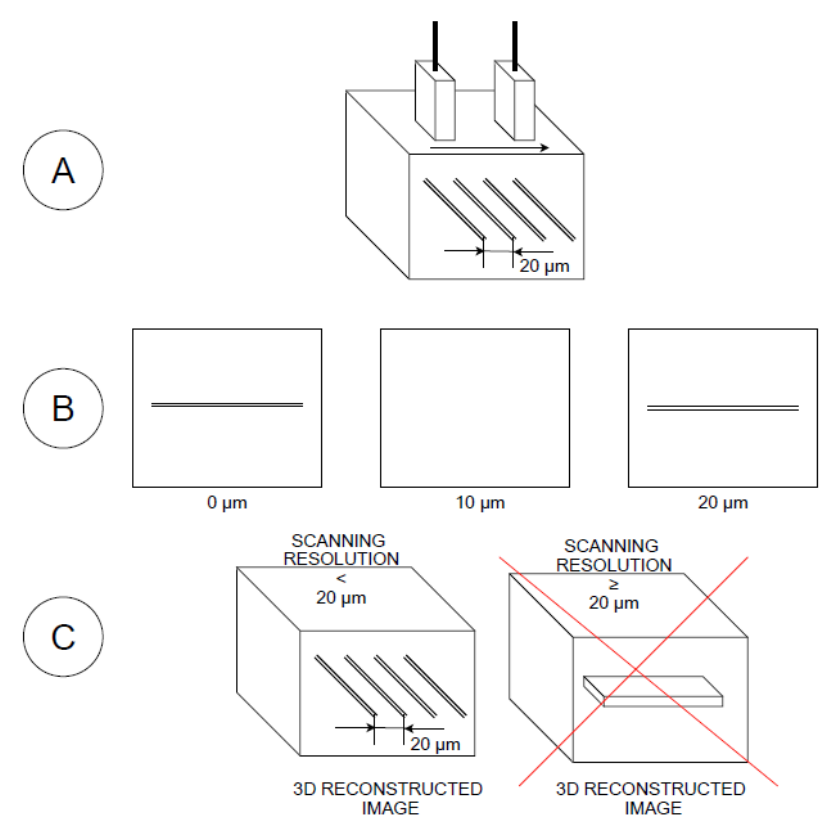

Fig. 11. Resolution Improvement by 3D Reconstruction

In order to do so, a dynamic scan with a shift of less than 20 $\mu \mathrm{m}$ is required to obtain the single lines. Here, the figure can be divided into three parts. Part A shows the phantom, B displays single images for a scanning resolution of $10 \mu \mathrm{m}$ and $\mathrm{C}$ shows the results for a successful and a failed reconstruction. The three images displayed below the phantom, show a shift of $10 \mu \mathrm{m}$. It can be seen that such a shift is sufficient to display the lines clearly as single structures. After reconstructing images with a shift of $10 \mu \mathrm{m}$ or even lower, an accurate representation of the phantom can be achieved.

\section{E. Panorama Scanning}

In the case, that the shift is equal or bigger than the distance between the lines, the $3 \mathrm{D}$ representation would be similar to the crossed representation, which is a wrong representation according to the phantom. However, such a shift needs to be highly accurate, which cannot be provided by a manual scan procedure. Therefore, on the basis of the previously shown results of the system, a possible application would also be the here introduced improvement of 2D ultrasound images on the basis of shift related $3 \mathrm{D}$ reconstruction. High resolution ultrasound data usually provides a limited field of view.
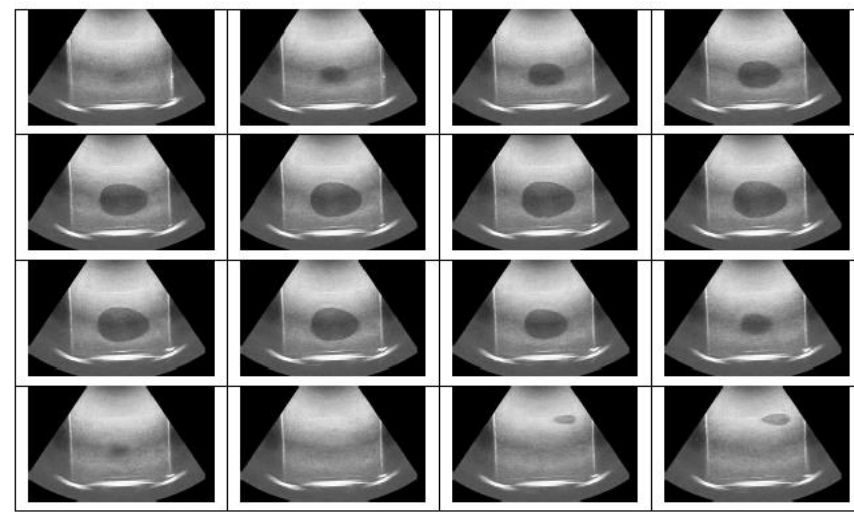

Fig. 12. Resolution Improvement by 3D Reconstruction.

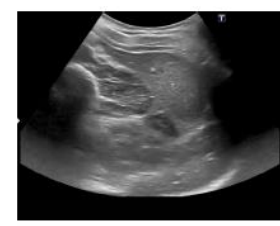

(a)

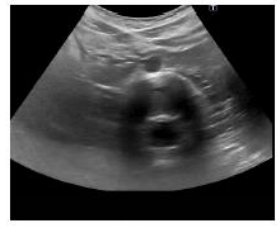

(b)

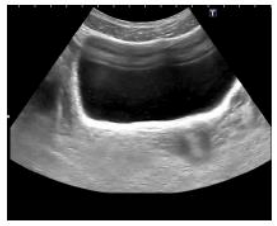

(c)
Fig. 13. Resolution Improvement by 3D Reconstruction.

For the visualization of larger volumes, a composition of the images is required. The most appropriate method to provide an extended field of view is panoramic scanning. Panoramas can be constructed by matching the images according to the location and orientation of the probe when it gets moved. The location of the probe and its motion are important parameters to subsequently display the anatomy in the same way it appears in nature. Therefore, the developed scanning system represents a suitable mechanism to provide the required accuracy in moving the probe. In order to test the performance of the system for providing panoramic scans, one phantom and a subject were scanned. For the phantom test 152 ultrasound slices have been acquired. The scanning resolution was set to $1 \mathrm{~mm}$ for a distance of $15 \mathrm{~cm}$. The time required was 4.05 seconds with a frame rate of $37 \mathrm{fps}$. The phantom represents a mimicking tissue object containing an egg shaped item. In Fig. 12, the coronal slices can be obtained. The object gets scanned from positive to negative $X$-direction. It displays its oval shape. Moreover, a smaller object is visible in the last two frames. It can be concluded at this point, that the system is able to scan short distances with low resolution in relatively short time. For the subject scan, three sequences of each 30.000 frames were acquired with a scanning resolution of $10 \mu \mathrm{m}$ during a scanning time of three times 14 minutes. The distance of the scanning area was defined as $30 \mathrm{~cm}$. The scanned medial and lateral tissues of the abdomen can be obtained in Fig. 13.

\section{F. 3D Reconstruction}

According to previous considerations, automatic systems are highly suitable for the improvement of resolution in ultrasound images. In order to obtain the images, the software of [33] was used and modified according to the phantom image characteristics. Therefore, functions had to be adjusted.

Here, the results of two approaches, being manual and automatic scanning, are compared after 3D reconstruction based on volume rendering. This can be obtained from Fig. 14. Fig. 14(a) shows the front view on the automatically acquired slides captured with coronal probe orientation. The interpolation results of two lines are displayed after sagittal cut Fig. 14(c). Fig. 14(b) represents the front view of the manually acquired frames captured with coronal probe orientation. After interpolation, the sagittal view on one line is shown in Fig. 14(d). For this purpose, a tissue mimicking phantom for lateral scanning resolution testing was used. For both setups a total of 1021 frames was captured within a time of 13.5 seconds per scan. The distance of the scanning area encloses $5 \mathrm{~cm}$. The scanning resolution of the system was set to $100 \mu \mathrm{m}$. In the case of automatic scanning, two lines of a diameter of $1 \mathrm{~mm}$ are displayed in their straight appearance after $3 \mathrm{D}$ reconstruction. The manual scan displays a line of 1 $\mathrm{cm}$ in diameter. 


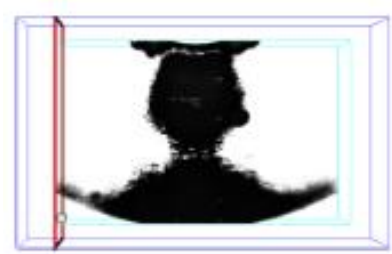

(a)

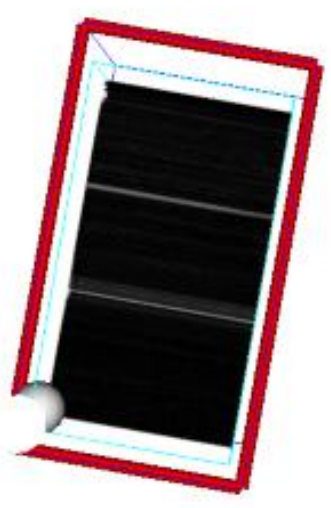

(c)

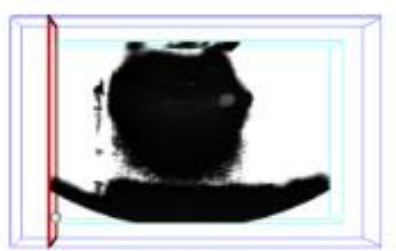

(b)

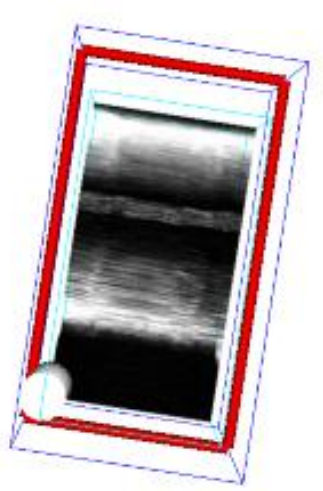

(d)
Fig. 14. Resolution Improvement by 3D Reconstruction: (a) shows the front view on the automatically acquired slides captured with coronal probe orientation, (b) represents the front view of the manually acquired frames captured with coronal probe orientation, (c) after sagittal cut of (a) and (d) sagittal cut of (b).

The white areas surrounding the line shall be ignored at this stage. Due to the fact that the data acquired by the use of freehand scanning includes variations in pressure and changing velocity, the line is shown irregularly after reconstruction. This may lead to misinterpretations of the actual structure. From these results, it can be concluded that manual scanning is highly unsuitable for accurate scan purposes and requires the substitution by a machine, like the automatic cervix scanning system.

\section{G. Application and Testing of Umbilicus Detection}

It is necessary to define an unambiguous initial point for the purpose of automatic scanning.
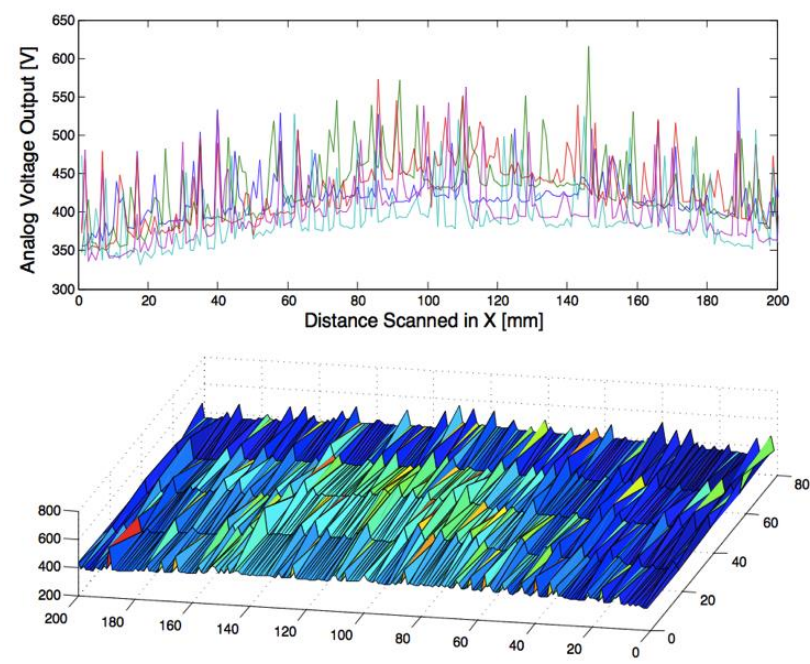

Fig. 15 2D (upper image) and 3D (lower image) plot of voltage output from IR sensor.
TABLE V

SCANNING PARAMETERS FOR SUBJECTS

\begin{tabular}{llllll}
\hline \hline & $\begin{array}{l}\mathrm{X}_{1 \mathrm{U}} \\
{[\mathrm{cm}]}\end{array}$ & $\begin{array}{l}\mathrm{X}_{2 \mathrm{U}} \\
{[\mathrm{cm}]}\end{array}$ & $\begin{array}{l}\mathrm{D}_{\mathrm{D}} \\
{[\mathrm{cm}]}\end{array}$ & $\begin{array}{l}\text { Total } \\
\text { Distance } \\
{[\mu \mathrm{m}]}\end{array}$ & $\begin{array}{l}\text { Time } \\
{[\mathrm{s}]}\end{array}$ \\
\cline { 2 - 6 } S1 & 4.5 & 16 & 0.3 & 205000 & 10.93 \\
S2 & 3.2 & 4.6 & 0 & 078000 & 4.16 \\
S3 & 7.7 & 41.9 & 0.5 & 226000 & 4.14 \\
S4 & 1.8 & 18 & -0.4 & 198000 & 10.65 \\
S5 & 2.4 & 5 & 0.1 & 074000 & 3.95 \\
S6 & -12.1 & 3 & -0.3 & 151000 & 8.05 \\
S7 & 7 & 5.1 & 0.6 & 261000 & 13.92 \\
S8 & 2.5 & 19.1 & 0.5 & 039000 & 2.18 \\
S9 & 13.2 & 15.1 & 0.2 & 157000 & 8.37 \\
S10 & 16.1 & 2.80 & 0.9 & 283000 & 15.10 \\
\hline \hline
\end{tabular}

The scan can be performed faster if the starting point is located close to the umbilicus area. Thus, the umbilicus was chosen to be the starting point of individual scan procedures. This setup resulted in a correct detection of the umbilicus in all cases with an accuracy of $95.9 \%$ for the $X$-plane and 98.4 $\%$ for the $Y$-plane. The voltage values from the microcontroller were plotted using MATLAB R2014a.

Spikes shown in the upper image of Fig. 15 are most probably caused by noise. Here, noise represents the impact of the surrounding light information like neon lamps used in the laboratory. In order to eliminate this undesired information, averaging was performed. After replacing the acquired data vector field by its average over time, an asymptotic approximation can be obtained. In the resulting curve a voltage output between 380 and 480 for one sequence can be seen. It displays the umbilicus marker at its highest point in 3D plot in Fig. 15 representing the highest voltage output recorded. This was performed in all cases.

\section{H. Umbilicus Detection Accuracy}

The accuracy of the umbilicus detection method is shown in Table VI. The parameters $X_{S}, X_{E}, Y_{S}$ and $Y_{E}$ refer to the start and end points in $X$ and $Y$-plane.

TABLE VI

RESULTS FOR AUTOMATIC SCANNING

\begin{tabular}{lllllllll} 
& $\begin{array}{l}\mathrm{X}_{\mathrm{S}} \\
{[\mathrm{cm}]}\end{array}$ & $\begin{array}{l}\mathrm{X}_{\mathrm{E}} \\
{[\mathrm{cm}]}\end{array}$ & $\begin{array}{l}\mathrm{E}_{\mathrm{X}} \\
{[\mathrm{cm}}\end{array}$ & $\begin{array}{l}\mathrm{A}_{\mathrm{x}} \\
{[\%]}\end{array}$ & $\begin{array}{l}\mathrm{Y}_{\mathrm{E}} \\
{[\mathrm{cm}]}\end{array}$ & $\begin{array}{l}\mathrm{E}_{\mathrm{Y}} \\
{[\mathrm{cm}]}\end{array}$ & $\begin{array}{l}\mathrm{E}_{\mathrm{Y}} \\
{[\mathrm{cm}]}\end{array}$ & $\begin{array}{l}\mathrm{A}_{\mathrm{Y}} \\
{[\%]}\end{array}$ \\
& & & & & & & & \\
S1 & 17.3 & 21.2 & 0.9 & 95.9 & 18.2 & 21.7 & 0.5 & 97.6 \\
S2 & 3.5 & 6.7 & 0.2 & 97.0 & 17.1 & 14.3 & 0.2 & 98.6 \\
S3 & 8.1 & 4.8 & 0.3 & 93.7 & 22.5 & 25.6 & 0.1 & 99.6 \\
S4 & 11.4 & 13.9 & 0.5 & 96.4 & 26.7 & 29.1 & 0.6 & 97.9 \\
S5 & 13.2 & 17.3 & 1.1 & 93.6 & 10.3 & 6.4 & 0.9 & 85.9 \\
S6 & 2.5 & 5.9 & 0.4 & 93.2 & 0.5 & 4.1 & 0.6 & 85.3 \\
S7 & 1.7 & 5.1 & 0.4 & 92.1 & 8.2 & 10.9 & 0.3 & 97.2 \\
S8 & 3.2 & 6.4 & 0.4 & 93.7 & 16.7 & 20.1 & 0.4 & 98.0 \\
S9 & 10.3 & 15.1 & 0.2 & 98.6 & 31 & 34.2 & 0.2 & 99.4 \\
S10 & 5.2 & 2.8 & 0.6 & 78.5 & 11 & 15.1 & 1.1 & 99.3 \\
\cline { 2 - 8 } & & & $\mathbf{0 . 5}$ & $\mathbf{9 5 . 9}$ & & & $\mathbf{0 . 4 9}$ & $\mathbf{9 8 . 4}$ \\
\hline
\end{tabular}


Errors have been observed, $5 \mathrm{~mm}$ in $X$-plane and $4 \mathrm{~mm}$ in $Y$-plane. This error refers to the axial correlation between marker and skin. It is the distance of the position of the transducer to the center of the umbilicus marker. The accuracy resulting for the $X$-plane is $95.9 \%$ and for the $Y$ plane is $98.4 \%$. These results show that the IR sensor approximates the umbilicus sufficiently high in order to perform abdominal scanning from this reference point.

This uncertainty of resolution in $X$-direction can be caused by light reflections of the skin during measurement or the fact, that human skin is close to the behavior of a black-body (Emissivity, $0.989 \pm 0.01$ ) [34] and therefore influences the axial accuracy. However, this approach can be replaced by a more accurate method in future if required. A resolution of 8000 pulses per rotation and a frequency of $50,000 \mathrm{~Hz}$ were set at this point. All sequences were started from the initial point of the probe with $X_{0}, Y_{0}$ and $Z_{0}$. The times, required for detecting the umbilicus, range from approximately 2 to 15 seconds, depending on the position of the subject and subsequently on the distance from the initial point to the umbilicus. The total distance in Table V refers to the distance, which is moved by the probe from the initial point to the umbilicus position. It can be obtained that currently only an unidirectional umbilicus detection method is used. The other distance measured is $D_{D}$ and represents the distance moved over marker before detection. Due to the previously discussed accuracy errors of around $5 \mathrm{~mm}$, the utilized method is subject to improvements.

\section{DISCUSSION}

A linear robot was used to visualize the human cervix and has proved to provide advantages over manual ultrasonography. This system has been compared with two other robotic ultrasound systems. The first system compared is a 6 DOF industrial robot for automatic ultrasound system for lower limb examination [35]. The system mainly encloses a robotic arm for accurate manipulation of ultrasound probe, a scanning system for generating ultrasound images of the scanned area and a workstation that converts all position and torque data to commands and showed good results for the clinical evaluation of vessels. The performance evaluation of this system shows that a position accuracy of $0.62 \pm 0.29 \mathrm{~mm}$ could be achieved in replay mode, which was $0.02 \mathrm{~mm}$ higher compared to teach mode results. The inter-distance accuracy for teach mode was measured $0.43 \pm 0.32 \mathrm{~mm}$, whereas for the replay mode, it was $0.42 \pm 0.33 \mathrm{~mm}$. Finally, the teach/replay repeatability calculated was $0.10 \pm 0.22 \mathrm{~mm}$.

The second system has been developed for automatic carotid artery examination. The systems main components are a master hand controller, a slave manipulator carrying the ultrasound probe and a computer control station. The operator remotely controls the probes position on the remote side. A resolution of $0.003 \mathrm{~mm}$ is reported here [36]. The linear robot tested in this paper allows a resolution up to $0.001 \mathrm{~mm}$ [37] and shows compared to both systems developed, the better performance.

Safety considerations were incorporated using initial point motions of the probe holder. However, this approach can get improved by the equipment of the holder using sensors in order to detect critical pressure.
Probe pressure must be applied evenly in order to get the accurate direction of the scan. Therefore, operator dependent scanning is less suitable than automatic for better image results concerning image quality. The pressure affects the echogenicity of the tissue and shortens the distance to the cervical structure. Moreover, image quality improvements were presented as low cost strategies in order to improve image resolution in ultrasound data. These include $3 \mathrm{D}$ image reconstruction using an already developed algorithm and the process of merging minimally shifted ultrasound image data. Furthermore, a precise panoramic scan of the abdomen was achieved using the linear robot in panorama scan modus offering a scanning length of $30 \mathrm{~cm}$ under constant pressure and with high movement resolution. This approach can be made autonomous by the usage of AI and control approaches allowing self-regulation by feedback information from sensors [38]. However, it is possible to consider the combination of the presented approaches with mobile applications [39] for procedure surveillance in terms of selfinterventions and quality control for the institute carrying out the specific procedure [40]. A clinical pathway may be created for personalized abdominal scanning [41] and online analysis using open source solutions [42]. However, results concerning specifically umbilicus detection in this paper have already been presented at the International Conference on Telecommunications and Signal Processing (TSP) [28].

\section{CONCLUSION}

A stable and precise method enabling personalized ultrasound scanning and automatic umbilicus detection has been successfully developed. Test results show that the system is able to capture ultrasound images of phantoms and subjects with an accuracy of $95.9 \%$ for the $X$-plane and 98.4 $\%$ for the $Y$-plane. Captured ultrasound images and positions can be used for the reconstruction of $2 \mathrm{D}$ and $3 \mathrm{D}$ panorama images for larger fields of view [43]. In order to improve the resolution of 2D panoramic view, it is recommended to use merging algorithms in combination with image registration and finite element modeling. However, it should be noted that this research does not aim at providing a ready to use device for clinical purposes since required guideline safety modalities were not considered at this point. Moreover, this study aims at improving existing ultrasound-based imaging methods by providing a motor-based scanning. Precise transducer probe movements allow accurate image capturing for subsequent processing of acquired data [44]. The analysis of ultrasound images containing abdominal structures allows a higher degree of operator independence. With this system, it is possible to perform automatic ultrasound cervix scanning with more comfort for the female patient focussing on biased cultural backgrounds as well as higher movement resolution and image quality. Furthermore, subject autonomy was achieved by defining acceptable pressure limits and the application of stop buttons for personal safety purposes [45]. This system will enable future operator independent task management [46], not only for obstetrics related diagnosis [47] but also for other internal organ imaging tools combined with E-Health platforms [48]. Therefore, it can be concluded that the presented system is a suitable low-cost approach for developing countries. 


\section{ACKNOWLEDGMENT}

The author would like to appreciate material support from Universiti Teknologi Malaysia as well as structural support from Technische Universität Ilmenau. Moreover, appreciation goes to anonymous reviewers for their constructive comments.

\section{REFERENCES}

[1] J. Ferlay, I. Soerjomataram, M. Ervik, R. Dikshit, S. Eser, C. Mathers, M. Rebelo, D. Parkin, D. Forman, F. Bray, "Cancer Incidence and Mortality Worldwide: IARC CancerBase No. 11," GLOBOCAN 2012 v1.0, International Agency for Research on Cancer, 2013.

[2] G. Lim, H. Yahaya, T. Lim, "The first report of the national cancer registry cancer incidence in Malaysia," Kuala Lumpur: Ministry of Health, pp. 57-58, 2003.

[3] D. Parkin, F. Bray, J. Ferlay, P. Pisani, "Global cancer statistics," CA: a cancer journal for clinicians, pp.74-108, 2005.

[4] C. Pahl, E. Supriyanto, "Personalized Cervix Ultrasound Scan Based On Robotic Arm," PSRC-Planetary Scientific Research Center Proceedings, 2012.

[5] C. Pahl, E. Supriyanto, N. Mahmood, J. Yunus, "Cervix Detection Using Squared Error Subtraction," Modelling Symposium (AMS), 2012 Sixth Asia. IEEE, pp. 121-125, 2012.

[6] A. I. B. Jeffree, C. Pahl, H. N. Abduljabbar, I. Ramli, N. I. B. Aziz, Y. M. Myint, E. Supriyanto, "Cervical Segmentation in Ultrasound Images using Level-set Algorithm," WSEAS International Conference on Biomedicine and Health Engineering, 2013.

[7] E. Supriyanto, J. Z. E. Soh, S. M. Nooh, "Myofascial Pain Syndrome, Trigger Point Detection based on Ultrasound Image," Proceedings of 15th WSEAS International Conference on Computers, pp.178-183, 2011.

[8] T. Wright Jr, M. Schiffman, D. Solomon, J. T. Cox, F. García, S. Goldie, D. Saslow, "Interim guidance for the use of human papillomavirus DNA testing as an adjunct to cervical cytology for screening," Obstetrics \& Gynecology, 2004, pp. 304-309.

[9] G. Brix, U. Lechel, G. Glatting, S. Ziegler, W. M. nzing, S. Müller, T. Beyer, "Radiation exposure of patients undergoing whole-body dualmodality 18F-FDG PET/CT examinations," Journal of Nuclear Medicine, pp. 608-613, 2005

[10] D. Frush, L. Donnelly, N. Rosen, "Computed tomography and radiation risks: what pediatric health care providers should know," Pediatrics, pp. 951-957, 2003.

[11] T. Slovis, "Children, computed tomography radiation dose, and the As Low As Reasonably Achievable (ALARA) concept," Pediatrics, pp.971-972, 2003.

[12] C. Pahl, A. Jeffree, Y. Myint, E. Supriyanto, "Cervix Segmentation in Ultrasound Data Based on Numerical Technique," International Journal of Biology and Biomedical Engineering, Issue 4, Volume 7, pp. 157-163, 2013.

[13] A. Fenster, D. Downey, H. Cardinal, "Three-dimensional ultrasound imaging," Physics in medicine and biology, 2001.

[14] R. Prager, U. Ijaz, A. Gee, G. Treece, "Three-dimensional ultrasound imaging," Proceedings of the Institution of Mechanical Engineers, Journal of Engineering in Medicine, pp.193-22, 2010.

[15] J. Szilard, "An improved three-dimensional display system," Ultrasonics, pp. 273-276, 1974.

[16] K. Pandey, A. Pradhan, A. Agarwal, A. Bhagoliwal, N. Agarwal, "Fluorescence Spectroscopy: A New Approach in Cervical Cancer," The Journal of Obstetrics and Gynecology of India, pp. 1-5, 2012.

[17] J. Henderson, M. Saraiya, G. Martinez, C. Harper, G. Sawaya, "Changes to cervical cancer prevention guidelines: Effects on screening among US women ages 15-29,“ Preventive Medicine, 2012

[18] C. Ravin, S. George, "Radiological Society of North America," Radiology, pp. 9-10, 2012.

[19] J. Rouyer, P. Lasaygues, S. Mensah, "Novel Ultrasound Tomograph for Anatomical Inspection," Acoustical Imaging, pp. 3-10, 2012.

[20] J. Charboneau, D. Levine, "Diagnostic ultrasound," JAMA, 2012.

[21] A. Wong, J. Scharcanski, "Monte Carlo despeckling of transrectal ultrasound images of the prostate," Digital Signal Processing, 2012.

[22] R. Rockland, H. Kimmel, J. Carpinelli, L. Hirsch, L. Burr-Alexander, "Medical Robotics in K-12 Education," Robots in K-12 Education, p. $120,2012$.

[23] P. N. Wells, "The safety of diagnostic ultrasound: report of a British Institute of Radiology Working Group,“" British Inst. of Radiology, 1987.

[24] W. R. Hedrick, D. L. Hykes," Biological Effects of Ultrasound I. Specification of Intensity," Journal of Diagnostic Medical Sonography, 7(4), pp. 188-193, 1991.
[25] P. N. Wells, "Ultrasonic imaging of the human body," Reports on progress in physics, 62(5), p. 671, 1999.

[26] E. Kimmel, "Cavitation bioeffects," Critical Reviews ${ }^{\mathrm{TM}}$ in Biomedical Engineering, 34(2), 2006.

[27] C. A. Speed, "Therapeutic ultrasound in soft tissue lesions." Rheumatology, 40(12), pp. 1331-1336, 2001

[28] C. Pahl, E. Supriyanto, "Infrared based clinical landmark determination for ultrasound image acquisition," In Telecommunications and Signal Processing (TSP), 2015 38th International Conference on, IEEE, pp. 236-240, 2015

[29] C. Pahl, E. Supriyanto, "Design of automatic transabdominal ultrasound imaging system," In Methods and Models in Automation and Robotics (MMAR), 2015 20th International Conference on, IEEE, pp. 435-440, 2015.

[30] C. Pahl, T.Y. Oon, E. Supriyanto, "Fuzzy system based face tracking for head movement control in progressive health care," In Methods and Models in Automation and Robotics (MMAR), 2015 20th International Conference on, IEEE, pp. 880-885, 2015.

[31] C. Pahl, E. Supriyanto, "Guide to fuzzy logic based transesophageal echocardiography motorized probe control," In Methods and Models in Automation and Robotics (MMAR), 2015 20th International Conference on, IEEE, pp. 892-896, 2015.

[32] W. E. Woodson, B. Tillman, P. Tillman, "Human factors design handbook: information and guidelines for the design of systems, facilities, equipment, and products for human use," 1992.

[33] K. W. Lai, Foetal abnormalities detection based on three dimensional nuchal translucency, Doctoral dissertation, Universiti Teknologi Malaysia, 2012.

[34] F. De Oliveira, S. Moreau, C. Gehin, A. Dittmar, "Infrared imaging analysis for thermal comfort assessment," Engineering in Medicine and Biology Society, 29th Annual International Conference of the IEEE, pp. 3373-3376, 2007.

[35] M. A. Janvier, L. G. Durand, M. H. R. Cardinal, I. Renaud, B. Chayer, P. Bigras, ... \& G. Cloutier, "Performance evaluation of a medical robotic 3D-ultrasound imaging system," Medical image analysis, 12(3), pp. 275-290, 2008.

[36] W. H. Zhu, S. E. Salcudean, S. Bachmann, P. Abolmaesumi, „Motion/force/image control of a diagnostic ultrasound robot," In Robotics and Automation, 2000. Proceedings. ICRA'OO. IEEE International Conference on, Vol. 2, pp. 1580-1585, 2000.

[37] C. Pahl, "Novel Method for Autonomous Ultrasound Cervix Scanning," Universiti Teknologi Malaysia, 2012.

[38] F. W. Lewis, S. Jagannathan, A. Yesildirak, "Neural network control of robot manipulators and non-linear systems," CRC Press, 1998.

[39] C. Pahl, M. Zare, L. Bordey, M. H. Satria, E. Supriyanto, Y. W. Hau, "Smartphone Applications in the Support of Weight Reduction and Goal Maintenance," Journal of Soft Computing and Decision Support Systems, 1(1), pp. 12-16, 2014

[40] M. Zare, C. Pahl, M. Nilashi, N. Salim, O. Ibrahim, "A Review of Semantic Similarity Measures in Biomedical Domain Using SNOMED-CT," Journal of Soft Computing and Decision Support Systems, 2(6), pp. 1-13, 2015.

[41] C. Pahl, E. Supriyanto, V. Detschew, "Evaluation of UML modeling tools for clinical pathways used in health information systems." In Information Systems and Technologies (CISTI), 2015 10th Iberian Conference on, IEEE, pp. 1-6, 2015.

[42] C. Pahl, N. B. Mohammad, E. Supriyanto, "Analysis on the electroretinography response for flickering and current stimulations." In Telecommunications and Signal Processing (TSP), 2015 38th International Conference on, IEEE, pp. 482-485, 2015

[43] J. Alcázar, M. Aubá, B. Olartecoechea, "Three-dimensional ultrasound in gynecological clinical practice," Reports in Medical Imaging, pp.1$13,2012$.

[44] C. Pahl, J. Lam, H. N. Abduljabbar, E. Supriyanto, Y. M. Myint "Performance Observation of Gabor Filter for Wall Thickness Measurement of Human Colon Based on Ultrasound Image," International Journal of Information and Electronics Engineering, 4(2), p. 171, 2014.

[45] K. Baker, V. Robertson, F. Duck, "A review of therapeutic ultrasound biophysical effects,” Physical Therapy, pp.1351-1358, 2001.

[46] C. Pahl, H.A.B Mazle, E. Supriyanto, ’Design of virtual instrument for automatic temperature visualization in magnetic fields using LabVIEW in combination with fiber-optical temperature measurement," In Automation, Robotics and Applications, pp. 393-399, 2015.

[47] C. Pahl, M. Zare, M. Nilashi, M.A. de Faria Borges, D. Weingaertner, V. Detschew, O. Ibrahim, E. Supriyanto, "Role of OpenEHR as an open source solution for the regional modelling of patient data in obstetrics." Journal of biomedical informatics, 55, pp. 174-187, 2015

[48] C. Pahl, et al., "Identification of Quality Parameters for an E-Health Platform in the Federal State of Thuringia in Germany." Journal of Soft Computing and Decision Support Systems 1.1, pp. 17-23, 2014. 